\title{
Central Nervous System Expression and PET Imaging of the Translocator Protein in Relapsing-Remitting Experimental Autoimmune Encephalomyelitis
}

\author{
Filomena Mattner ${ }^{1,2}$, Maria Staykova ${ }^{3,4}$, Paula Berghofer ${ }^{1}$, Heng Jian Wong ${ }^{3}$, Susan Fordham ${ }^{3}$, Paul Callaghan ${ }^{1}$, \\ Timothy Jackson ${ }^{1}$, Tien Pham ${ }^{1}$, Marie-Claude Gregoire ${ }^{1}$, David Zahra ${ }^{1}$, Gita Rahardjo ${ }^{1}$, David Linares ${ }^{3}$, \\ and Andrew Katsifis ${ }^{2}$ \\ ${ }^{1}$ Life Sciences Division, Australian Nuclear Science and Technology Organisation, Lucas Heights, New South Wales, Australia; \\ ${ }^{2}$ Department of PET and Nuclear Medicine, Royal Prince Alfred Hospital, Camperdown, New South Wales, Australia; ${ }^{3}$ Neurosciences \\ Research Unit, The Canberra Hospital, Canberra, Australian Capital Territory, Australia; and ${ }^{4}$ The John Curtin School of Medical \\ Research, The Australian National University, Canberra, Australian Capital Territory, Australia
}

Glial neuroinflammation is associated with the development and progression of multiple sclerosis. PET imaging offers a unique opportunity to evaluate neuroinflammatory processes longitudinally in a noninvasive and clinically translational manner. ${ }^{18} \mathrm{~F}-$ PBR111 is a newly developed PET radiopharmaceutical with high affinity and selectivity for the translocator protein (TSPO), expressed on activated glia. This study aimed to investigate neuroinflammation at different phases of relapsing-remitting (RR) experimental autoimmune encephalomyelitis (EAE) in the brains of SJL/J mice by postmortem histologic analysis and in vivo by PET imaging with ${ }^{18} \mathrm{~F}-\mathrm{PBR} 111$. Methods: RR EAE was induced by immunization with PLP $_{139-151}$ peptide in complete Freund's adjuvant. Naive female SJL/J mice and mice immunized with saline-complete Freund's adjuvant were used as controls. The biodistribution of ${ }^{18} \mathrm{~F}-\mathrm{PBR} 111$ was measured in 13 areas of the central nervous system and compared with PET imaging results during different phases of RR EAE. The extents of TSPO expression and glial activation were assessed with immunohistochemistry, immunofluorescence, and a real-time polymerase chain reaction. Results: There was significant TSPO expression in all of the central nervous system areas studied at the peak of the first clinical episode and, importantly, at the preclinical stage. In contrast, only a few TSPO-positive cells were observed at the second episode. At the third episode, there was again an increase in TSPO expression. TSPO expression was associated with microglial cells or macrophages without obvious astrocyte labeling. The dynamics of ${ }^{18} \mathrm{~F}-\mathrm{PBR} 111$ uptake in the brain, as measured by in vivo PET imaging and biodistribution, followed the pattern of TSPO expression during RR EAE. Conclusion: PET imaging with the TSPO ligand ${ }^{18} \mathrm{~F}-\mathrm{PBR} 111$ clearly reflected the dynamics of microglial activation in the SJL/J mouse model of RR EAE. The results are the first to highlight the discrepancy between the clinical symptoms of EAE and TSPO expression in the brain, as measured by PET imaging at the peaks of various EAE episodes. The results suggest a significant role for

Received May 31, 2012; revision accepted Sep. 14, 2012.

For correspondence or reprints contact: Filomena Mattner, Department of PET and Nuclear Medicine, Prince Alfred Hospital, B63, Level A7, Missenden

Rd, Camperdown, NSW 2050, Australia.

E-mail: mattnerf@gmail.com

Published online Jan. 15, 2013.

COPYRIGHT @ 2013 by the Society of Nuclear Medicine and Molecular Imaging, Inc.
PET imaging investigations of neuroinflammation in multiple sclerosis and allow for in vivo follow-up of antiinflammatory treatment strategies.

Key Words: translocator protein; PET; inflammation; multiple sclerosis; experimental autoimmune encephalomyelitis

J Nucl Med 2013; 54:291-298

DOI: 10.2967/jnumed.112.108894

$\mathbf{M}$ ultiple sclerosis (MS), a severe disorder of the central nervous system (CNS), is characterized by inflammation, myelin loss, gliosis, varying degrees of axonal degeneration, and oligodendrocyte pathology (1). The majority of MS patients experience relapsing-remitting (RR) symptoms followed by a secondary progressive phase leading to permanent disability once chronic disease has set in.

Consequently, experimental autoimmune encephalomyelitis (EAE), an animal model of MS, provides a powerful tool for investigating the pathogenesis of MS. Immunization of several rodent strains with various neuroantigens has resulted in the induction of different types of EAE (2-4) that correspond to different phases of the human disease (5). Because of the variable inflammation, demyelination, remyelination, glial proliferation, and neurodegeneration in the CNS white matter and gray matter, information (obtained noninvasively) about the early symptoms and diagnosis of MS, disease progression, and responses to various treatments would be beneficial for MS management.

In addition to conventional radiologic methods (6-8), noninvasive molecular imaging modalities (PET and SPECT) have gained importance as tools for the in vivo assessment of neuroinflammation. Significantly, upregulation of the translocator protein (TSPO) (9) is considered to be a hallmark of neuroinflammation.

In a healthy brain, the TSPO is expressed at low levels on astrocytes and resting microglial cells (10), where it is 
mostly involved in steroidogenesis (11) and mitochondrial function (12). However, its levels are markedly increased during neuroinflammation and neurodegeneration. Consequently, it has been suggested that TSPO functions may be important in modulating neuronal damage (12) and hence may support accelerated microglial proliferation. Furthermore, because TSPO expression in healthy subjects is not detectable, quantitative PET imaging provides an excellent opportunity to image the early symptoms of diseases through the use of radiotracers that target the TSPO (13-15).

The TSPO ligand ${ }^{11} \mathrm{C}-\mathrm{PK} 11195$ was one of the first PET ligands used for the imaging of activated microglia in various neurodegenerative diseases (16), and several clinical studies have been performed $(17,18)$. Although ${ }^{11} \mathrm{C}-(R)$-PK11195 has been widely used, its high levels of plasma protein binding, high levels of nonspecific binding, relatively poor blood-brain barrier permeability, and short half-life limit its use in routine brain imaging (19). This fact has prompted the development of alternative PET radioligands for the TSPO, including analogs bearing the longer-lived ${ }^{18} \mathrm{~F}$. The imidazopyridine ${ }^{18}$ F-PBR111 (20) is a high-affinity, specific radiotracer for the TSPO with excellent in vivo characteristics for use as an imaging neuroinflammation biomarker $(20,21)$.

The aim of this project was to investigate brain neuroinflammation with postmortem histologic analysis and in vivo PET/CT with ${ }^{18} \mathrm{~F}-\mathrm{PBR} 111$ at different phases of RR EAE in the SJL/J mouse model.

\section{MATERIALS AND METHODS}

\section{Radiotracer}

${ }^{18} \mathrm{~F}-\mathrm{PBR} 111$ was prepared on the Tracerlab FXFN modules (GE Healthcare) as described elsewhere (22). The product was formulated to a concentration of $20 \mathrm{MBq} / 100 \mu \mathrm{L}$ in saline containing less than $1 \%$ ethanol. The specific activity of ${ }^{18} \mathrm{~F}-\mathrm{PBR} 111$ was $150-400 \mathrm{GBq} / \mu \mathrm{mol}$. For biodistribution and competition studies, the product was diluted to $0.70 \mathrm{MBq} / 100 \mu \mathrm{L}$. For imaging studies, ${ }^{18} \mathrm{~F}-\mathrm{PBR} 111$ was formulated to $0.06 \mathrm{nmol} / 100 \mu \mathrm{L}$ of PBR111.

\section{Animals}

Female SJL/J mice were obtained from the Animal Resource Centre (Perth, Western Australia, Australia). They were housed in groups of 2 or 3 under a 12-h light-dark cycle in a temperatureand humidity-controlled environment. Food and water were freely available. This study was approved by the ANSTO or Australian Nuclear Science and Technology Organization Animal Ethics Committee, and all procedures complied with the Australian Code of Practice for the Care and Use of Animals for Scientific Purposes.

\section{RR EAE}

The mice $(n=75)$ were immunized subcutaneously in both flanks with $100 \mu \mathrm{L}$ of an emulsion containing $50 \mu \mathrm{L}$ of PLP $_{139-151}$ peptide (50 $\mu \mathrm{g}$ of $\mathrm{PLP}_{139-155}$ peptide carboxamide, HSLGKLGHPDKF-NH $\mathrm{N}_{2}$ (PLP) and $50 \mu \mathrm{L}$ of complete Freund's adjuvant (Mycobacterium tuberculosis $\mathrm{H} 37 \mathrm{RA}$ at $8.3 \mathrm{mg} / \mathrm{mL}$ and Mycobacterium butyricum at $0.5 \mathrm{mg} / \mathrm{mL}$ ) (CFA). The mice were examined daily, and a neurologic score was assigned according to the following scale: 0 , asymptomatic; 0.5 , tip of the tail flaccid; 1 , distal half of the tail flaccid; $1.5,3 / 4$ of the tail flaccid; 2 , entire tail flaccid; 2.5 , ataxia and difficulty righting; 3 , weakness of hind limbs; and 3.5, dragging of hind feet. The body weight was also monitored, and the clinical stages of EAE were assigned according to neurologic signs and weight loss as follows: preclinical, no neurologic symptoms and no weight loss; episodes, weight loss and neurologic symptoms; recovery from episodes, decrease in neurologic symptoms and increase in body weight. Two types of controls were used: naive mice $(n=11)$ and mice immunized with saline-CFA $(n=14)$.

\section{Biodistribution}

The mice were assessed at different EAE stages: preclinical (score of $0, n=6$ ), first episode (scores of 1.5-3.5, $n=9$ ), recovery from first episode (1-2 d at score of $0, n=4)$, full recovery from first episode (more than $10 \mathrm{~d}$ at score of $0, n=$ 8 ), and second episode (scores of $0.5-3.5, n=6$ ). Naive mice $(n=5)$ and mice immunized with saline-CFA (13 d after immunization, $n=5$ ) served as controls. The animals were injected via the tail vein with $0.70 \mathrm{MBq}$ of ${ }^{18} \mathrm{~F}-\mathrm{PBR} 111$ in $0.1 \mathrm{~mL}$ of saline. Tissue samples obtained from the CNS (olfactory bulbs, frontal cortex, posterior cortex, hippocampus, hypothalamus, midbrain, cerebellum, medulla oblongata, medulla pons, cervical enlargement, thoracic spinal cord, and lumbar spinal cord), peripheral organs (heart, lungs, and kidneys), and blood were removed $1 \mathrm{~h}$ later, weighed, and the radioactivity was measured with an automated $\gamma$-counter. The percentage injected dose was calculated by comparison with a diluted standard solution derived from the injected solution. Radioactivity concentrations were expressed as the percentage injected dose/g of wet tissue.

\section{Competition Studies}

The in vivo specificity of ${ }^{18} \mathrm{~F}-\mathrm{PBR} 111$ was evaluated in 10 mice at the first EAE episode (clinical scores of 2-2.5). Five mice were injected with PK11195 (1 mg/kg; dissolved in saline containing $5 \%$ dimethyl sulfoxide) 5 min before the injection of $0.70 \mathrm{MBq}$ of the radiotracer ${ }^{18} \mathrm{~F}-\mathrm{PBR} 111$. Uptake in the brain and peripheral organs was compared with that in mice $(n=5)$ injected with ${ }^{18} \mathrm{~F}$ PBR111 only. The mice were sacrificed $1 \mathrm{~h}$ later, and tissue samples were recovered for analysis. Radioactivity concentrations were calculated as described earlier.

\section{PET Imaging}

Longitudinal imaging and displacement studies were performed on an Inveon (Siemens) small-animal PET/CT imaging system. For ${ }^{18}$ F-PBR111 distribution, 12 mice immunized with PLP-CFA were imaged 6 times over a period of 35-41 d. Imaging was performed before immunization with PLP-CFA for baseline comparisons and on days $6,13,20,27,35$, and 41 after immunization. Therefore, the numbers of animals at different clinical stages of EAE were as follows: preclinical (score of $0, n=4$ ), first episode (scores of 1.5-3.5, $n=8$ ), recovery from first episode (score of 0.5, $n=3$ ), recovery from first episode (3-7 d at score of $0, n=5$ ), full recovery from first episode (more than $10 \mathrm{~d}$ at score of $0, n=5$ ), second episode (scores of 1.5-3.5, $n=3$ ), recovery from second episode (score of $1, n=2$ ), recovery from second episode (3-10 d at score of $0, n=2$ ), and third episode (scores of 3-3.5, $n=2$ ). A group of control mice given saline-CFA $(n=4)$ and a group of naive mice $(n=6)$ were imaged 3 or 4 times each during the same period.

The mice were injected via the tail vein with ${ }^{18}$ F-PBR111 (0.06 nmol) and anesthetized with isoflurane (in medical oxygen; induction, 4.5\%; maintenance, $1 \%-3 \%$ ) 20 to $30 \mathrm{~min}$ later for PET 
imaging. The body temperature was maintained with a feedbackregulated heating pad. One or 2 mice were placed with their heads carefully positioned at the center of the field of view of the PET/CT scanner, and physiologic parameters (heart rate, respiration, and body temperature) were monitored (BioVet; $\mathrm{m} 2 \mathrm{~m}$ Imaging Corp.) for the entire scanning period. To avoid movement, the mouse was restrained with a webbed sock and adhesive tape. Lacri-lube (Allergan) was placed in the mouse's eyes to prevent drying while the mouse was anesthetized.

A CT scan was conducted for 9 min on each animal (for atlas anatomic information), and then a PET acquisition was conducted for $10 \mathrm{~min}$ (40 $\mathrm{min}$ after injection of $\left.{ }^{18} \mathrm{~F}-\mathrm{PBR} 111\right)$. This optimal time point was selected on the basis of preliminary studies in which animals were imaged over a period of $2 \mathrm{~h}$. After each imaging study, the mouse was removed from the animal bed and placed in a warm cage to recover from anesthesia.

The displacement of ${ }^{18} \mathrm{~F}-\mathrm{PBR} 111$ was evaluated $13 \mathrm{~d}$ after immunization in 10 mice, 5 of which were immunized with PLP-CFA (at the first episode; clinical scores of 2-2.5) and 5 of which were immunized with saline-CFA (controls). The mice were anesthetized with isoflurane, positioned in the PET/CT scanner as described earlier, and injected via the tail vein with ${ }^{18}$ F-PBR111 (0.06 nmol); scanning commenced simultaneously with the injection. After $50 \mathrm{~min}$ of imaging, PBR111 (1 mg/kg in $2 \%$ acetic acid-saline) was injected, and imaging was continued to $100 \mathrm{~min}$. The mice were sacrificed by $\mathrm{CO}_{2}$ overdose, and their brains and spinal cords were removed and frozen in liquid nitrogen for immunochemistry or treated with triazole for a realtime (RT) polymerase chain reaction (PCR). Two mice that reached a clinical score of 3.5 and did not show remission over $24 \mathrm{~h}$ were sacrificed before the end of the longitudinal study, and their brains were removed for RT PCR.

\section{PET Data Analysis}

All PET data were reconstructed with an ordered-subset expectation maximization 3-dimensional maximum a posteriori algorithm (5 ordered-subset expectation maximization 3-dimensional iterations with 16 subsets, followed by 10 maximum a posteriori iterations; $\beta=$ 0.1) (23). The dynamic PET data were converted into histograms in 30 frames $(4 \times 30 \mathrm{~s}, 4 \times 120 \mathrm{~s}, 7 \times 300 \mathrm{~s}, 1 \times 240 \mathrm{~s}, 7 \times 30 \mathrm{~s}, 4 \times 120$ s, $3 \times 600 \mathrm{~s}$ ) before reconstruction. PET and CT volumes were automatically coregistered for each of the scans. All CT volumes for the same mouse were manually coregistered to the first one (reference) with image visualization and processing software (24), and a mouse brain atlas (25) was finally coregistered to the reference. Regions of interest from the atlas were used to compute statistics. Data were converted to activity concentrations $(\mathrm{kBq} / \mathrm{mL})$ with calibration factors.

\section{RT PCR}

The olfactory bulbs, cerebellum, medulla oblongata, and lumbar spinal cords were collected from naive animals $(n=3)$, from animals immunized with saline-CFA $(n=3)$, and at the following EAE stages: first episode $(n=3)$, second episode $(n=3)$, and third episode $(n=1)$. The tissue samples were kept in triazole at $-80^{\circ} \mathrm{C}$. Total RNA was prepared according to the instructions of the manufacturer (Invitrogen). The purity of RNA was determined by the absorption by $260 \mathrm{~nm}$ divided by the absorption at $280 \mathrm{~nm}$. RNA $(5 \mu \mathrm{g})$ for each sample was reverse transcribed into complementary DNA with an Omniscript RT kit (Qiagen). RT PCR based on SYBR Green (Qiangen) was used to measure relative TSPO gene expression. Each sample was assayed in duplicate with the $\beta$-actin-specific primers that were used as an internal standard. Negative controls lacking the complementary DNA template were run with every assay to assess specificity. The mouse TSPO primer sets used in this study were (R) 5'-TCC CAG CTC TTT CCA GAC TAT GT and (F) 5'-TGG TAT GCT AGC TTG CAG AAA CC. PCR amplification was performed in a PCR system (Applied Biosystems). Relative quantification with the comparative threshold $(\mathrm{Ct})$ method was used for analysis. The results were expressed as fold change relative to the values for controls.

\section{Immunohistochemistry and Immunofluorescence}

Brain TSPO expression was visualized by immunohistochemistry in 3 naive mice (controls) and at the first (score of 2.5, $n=3$ ), second (score of 2.5,n=3), and third (score of 3, n= 1) EAE episodes. Air-dried 18- $\mu \mathrm{m}$ cryostat sections were fixed in cold $\left(-15^{\circ} \mathrm{C}\right)$ acetone for $4 \mathrm{~min}$. After incubation at room temperature with $\mathrm{Fc}$ receptor blocking for $30 \mathrm{~min}$ and with $5 \%$ bovine serum albumin in phosphate-buffered saline for $1 \mathrm{~h}$, the sections were incubated with an anti-TSPO antibody (goat anti-human, rat, and mouse antibody; W-12; Santa Cruz Biotechnology) overnight at $4^{\circ} \mathrm{C}$. The secondary antibody was horseradish peroxidase-labeled rabbit anti-goat IgG (Santa Cruz Biotechnology), and peroxidase development was done with a Vector Laboratories kit (Vectastain DAB).

For immunofluorescence studies, blocking and labeling with the anti-TSPO antibody were done as described earlier. Astrocytes were labeled with rabbit polyclonal anti-glial fibrillary acidic protein (GFAP) antibody (Sapphire). The secondary antibody for the TSPO was phycoerythrin-labeled anti-goat IgG (Santa Cruz Biotechnology), and that for the GFAP was fluorescein isothiocyanate-labeled anti-rabbit IgG. Fluorescein isothiocyanate-labeled anti-mouse macrophage F4/80 (clone A3-1; BioLegend) and fluorescein isothiocyanate-labeled anti-mouse $\mathrm{CD} 11 \mathrm{~b}$ (BD Biosciences Pharmingen) antibodies were used to visualize activated macrophages or microglial cells. Corresponding sera and antibodies were used as controls. Fluorescence was examined with confocal microscope Leica TCS SP5 (Leica Microsystems).

\section{Statistical Analysis}

Statistical analysis was performed with Prism v5.0 (GraphPad Software Inc.). Values for control mice and mice immunized with PLP-CFA were compared by use of a one-way ANOVA followed by a Tukey or Dunnett post hoc test. A $P$ value of less than 0.05 was considered significant. Data are expressed as mean $\pm \mathrm{SD}$.

\section{RESULTS \\ RR EAE}

Of the mice immunized with PLP-CFA $(n=75), 90 \%$ developed clinical EAE. The signs of the first episode occurred at approximately day 12 , and all mice recovered from the first episode. Of the mice monitored for $35 \mathrm{~d}, 50 \%$ relapsed to a second episode, and $6 \%$ relapsed to a third episode.

A representation of the course of EAE (monophasic or RR), based on changes in neurologic symptoms and body weight, is shown in Figure 1. 
FIGURE 1. Clinical course of EAE induced by PLP-CFA in SJL/J mice.

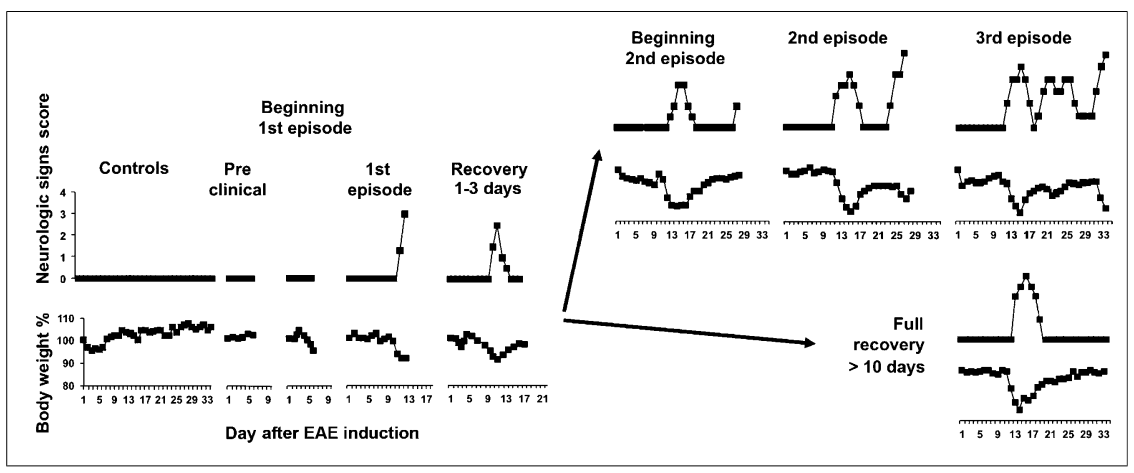

\section{Biodistribution of and Competition with ${ }^{18} \mathrm{~F}-\mathrm{PBR} 111$ in Mice with EAE}

All groups of animals showed similar levels of uptake of ${ }^{18}$ F-PBR111 in peripheral tissues. The disease severity had no influence on ${ }^{18} \mathrm{~F}-\mathrm{PBR} 111$ uptake or distribution in peripheral tissues. Because the levels of CNS uptake of ${ }^{18} \mathrm{~F}-\mathrm{PBR} 111$ in the 2 types of controls (naive mice and mice immunized with saline-CFA) were not significantly different, the results (data not shown) were combined and used for comparison with the results for mice with EAE.

The uptake of radioactivity in the CNS reflected the clinical stage of the inflammatory process because there was a statistically significant difference $(P<0.05)$ between mice with clinical signs of EAE (independently of the episode) and controls (Fig. 2A). In the preclinical group $(n=6)$, there was a significant increase in radioactivity in all areas of the CNS (60\%-100\%) above the control values. The olfactory bulbs, cerebellum, and hippocampus responded first to the autoimmune inflammation, as indicated by the increased uptake of ${ }^{18} \mathrm{~F}-\mathrm{PBR} 111$ in mice that were not yet showing any decrease in body weight $(n=2)$. The increase escalated to $100 \%-500 \%$ at the beginning of the first episode and reached $300 \%-1,100 \%$ at its peak. The clinical severity of EAE was reflected by an increase in the amount of ligand bound to all

FIGURE 2. Uptake of ${ }^{18} \mathrm{~F}-\mathrm{PBR} 111$ in CNS. (A) Uptake in CNS of mice immunized with PLP-CFA. (Inset) Correlation between ${ }^{18} \mathrm{~F}$ PBR111 uptake and clinical score at first EAE episode $(n=6-9)$. (B) Uptake in CNS at recovery from first EAE episode $(n=4-8)$. ${ }^{\star} P<0.05$ (vs. control). \%ID = percentage injected dose.

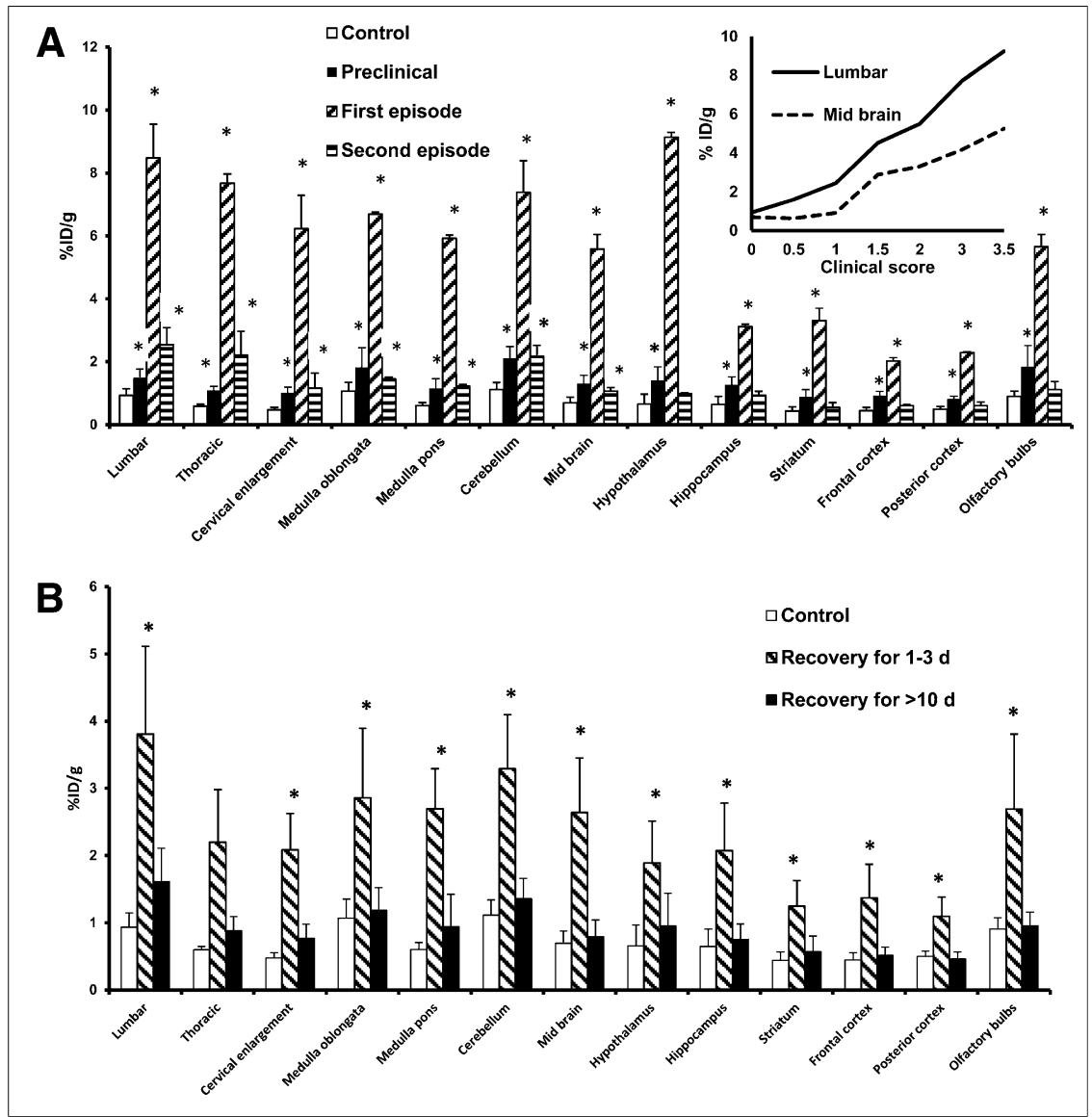


CNS areas and was positively correlated with the clinical scores at the first episode (Fig. 2A, inset).

Interestingly, compared with the findings for the first episode, there was a modest increase in CNS ${ }^{18} \mathrm{~F}-\mathrm{PBR} 111$ uptake at the second episode (50\%-300\%). The lumbar spinal cord and thoracic spinal cord showed the largest increase (150\%-300\%), whereas the hippocampus, striatum, frontal cortex, posterior cortex, and olfactory bulbs were not affected at the second episode. The increase in radioactivity in the medulla oblongata, cerebellum, midbrain, and hypothalamus was between $40 \%$ and $100 \%$ (Fig. 2A).

After the first episode, as animals recovered (weight gain and decrease in neurologic deficits; $1-3 \mathrm{~d}$ at score of 0 ), the uptake of ${ }^{18} \mathrm{~F}-\mathrm{PBR} 111$ in the CNS decreased by $50 \%$, first in the posterior cortex and then in the frontal cortex and striatum. In mice that regained their initial body weight and did not enter a second episode, the cerebellum and spinal cord were the last to return to normal. When the animals were at score 0 for more than $10 \mathrm{~d}$, the radioactivity in all CNS areas was similar to the control values (Fig. 2B).

The administration of PK11195 before the injection of the radiotracer reduced the uptake of ${ }^{18} \mathrm{~F}$-PBR111 in the CNS by $50 \%-80 \%$ (Fig. 3), confirming the specificity of ${ }^{18}$ F-PBR111 binding.

\section{PET Imaging}

The feasibility of using the TSPO as a marker to assess the degree of glial activation in living mice was evaluated with ${ }^{18} \mathrm{~F}-\mathrm{PBR} 111$ on a small-animal PET/CT imaging system. The average ${ }^{18} \mathrm{~F}-\mathrm{PBR} 111$ uptake (expressed as the percentage injected dose $\pm \mathrm{SD} / \mathrm{mL}$ ) in the brain before immunization with PLP-CFA and in naive controls (6 animals imaged 20 times; percentage injected dose, $1.4 \pm$ $0.2 / \mathrm{mL}$ ) was the same as the average uptake in controls immunized with saline-CFA and imaged during the 35-d study (4 animals imaged 12 times; percentage injected dose, $1.5 \pm 0.1 / \mathrm{mL}$ ). The peak average increase in radioactivity in the whole brain, $135 \% \pm 20 \%$, was reached at

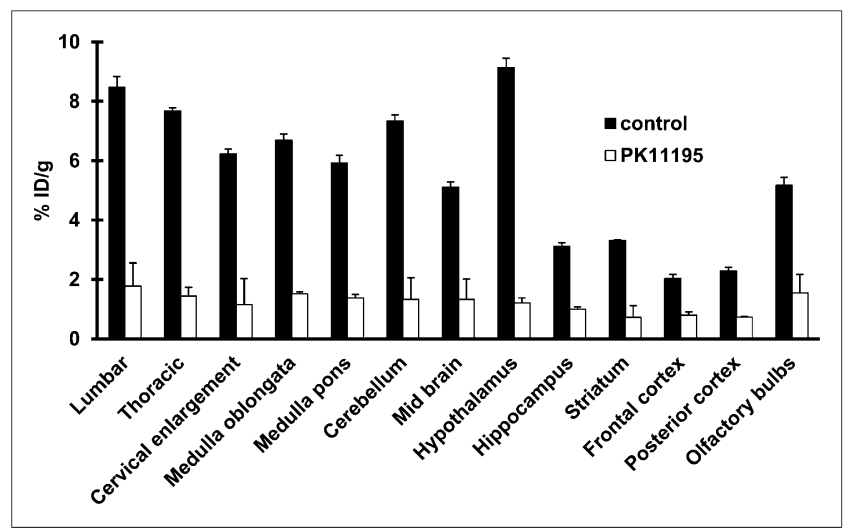

FIGURE 3. Blocking of ${ }^{18} \mathrm{~F}$-PBR111 uptake by PK11195 in CNS of mice at first EAE episode, with clinical scores of 2-2.5 $(n=5)$. ${ }^{*} P<$ 0.05 (vs. control). \%ID = percentage injected dose.

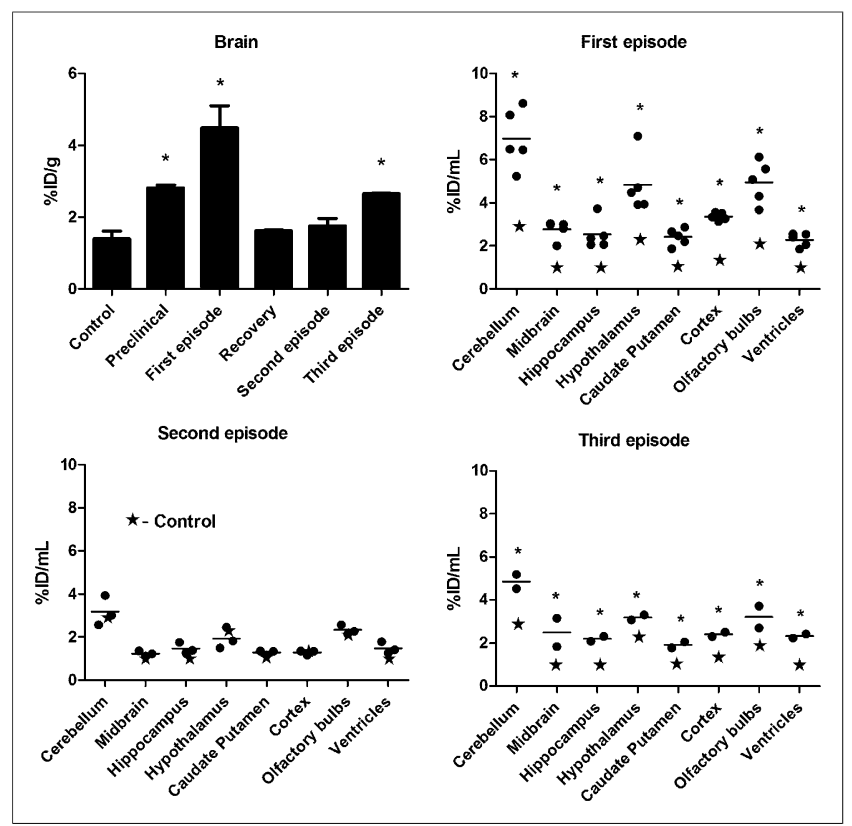

FIGURE 4. Uptake of ${ }^{18} \mathrm{~F}-\mathrm{PBR} 111$ in CNS, as measured by PET. Brain regions at different disease stages are shown $(n=2-5)$ : first episode, with EAE scores of 2-2.5 $(n=5)$; second episode, with EAE scores of 1.5-3.5 $(n=3)$; and third episode, with EAE scores of 3-3.5 $(n=2)$. ${ }^{\star} P<0.05$ for control vs. EAE. \%ID = percentage injected dose.

the first episode and was followed by a return to almost baseline values $(5 \%)$ in animals with a clinical score of 0 for more than $10 \mathrm{~d}$ and no relapse (Fig. 4). Analysis of the PET images from 8 brain areas confirmed the significant difference among the 3 EAE episodes (Fig. 4). The region of the ventricles was also included, and this region exhibited the same dynamics as the others studied.

The uptake of ${ }^{18} \mathrm{~F}-\mathrm{PBR} 111$ in the brain, as assessed by the longitudinal PET imaging study during the course of the disease, showed a large increase in the uptake of activity at the first episode and a decrease at recovery. In agreement with the biodistribution data, the increase in the uptake of activity at the second episode was not significant, but a significant increase in uptake occurred at the third episode.

The specificity of the TSPO ligand ${ }^{18}$ F-PBR 111 was demonstrated in a PET displacement study in which PBR111 was injected 50 min after the administration of the radiotracer (Fig. 5). A marked reduction in the radioactivity in the peripheral organs expressing the TSPO and in the brain $(39.7 \% \pm 3.3 \% ; n=5 ; P<0.05)$ was observed. Figure 5 also shows an example of the specificity of ${ }^{18}$ F-PBR111 during PET imaging in the brain.

\section{Immunohistochemistry and RT PCR}

To corroborate the uptake of ${ }^{18} \mathrm{~F}-\mathrm{PBR} 111$ with TSPO expression (as protein and messenger RNA) in different brain structures during the EAE episodes, we performed immunohistochemistry and RT PCR (Fig. 6). By immunohistochemistry, numerous cells were found TSPO positive during the first EAE episode, whereas only a few positive 
cells were observed during the second episode. The number of TSPO-positive cells increased during the third episode (Fig. 6A). The dynamics of TSPO expression measured as protein closely corresponded to the expression of TSPO messenger RNA in the 4 analyzed CNS structures (olfactory bulbs, cerebellum, medulla oblongata, and lumbar spinal cord) in mice during the first, second, and third EAE episodes (Fig. 6B). TSPO expression increased significantly $(P<0.5)$ in the 4 regions only during the first EAE episode.

\section{Immunofluorescence}

The large differences in the CNS uptake of the TSPO ligand ${ }^{18}$ F-PBR111 during 3 EAE episodes warranted the study of cells expressing the TSPO. Double staining for astrocytes (GFAP positive) and the TSPO and double staining for activated macrophages or microglial cells (F4/80 and CD11b positive) and the TSPO were performed on brain cryosections. Figure 7 shows a good correlation between the labeling of activated macrophages or microglial cells and TSPO expression. The intense labeling of activated macrophages or microglial cells during the first episode and the weaker labeling during the third episode paralleled the significantly high level of ${ }^{18} \mathrm{~F}-\mathrm{PBR} 111 \mathrm{up}-$ take during the first episode and the lower level of ${ }^{18} \mathrm{~F}$ PBR111 uptake during the third episode. Only single cells appeared positive for both-F4/80 and the TSPO-during the second episode. In all the regions assessed, astrocytes (GFAP-positive cells) were TSPO negative.

\section{DISCUSSION}

In previous work, we monitored TSPO expression in rats with monophasic EAE induced by myelin basic proteinCFA (26) and transferred EAE induced by myelin basic protein-specific T lymphoblasts (27) as well as in a model of demyelination induced by cuprizone (28). In vitro and in vivo analyses with the SPECT TSPO ligand ${ }^{123}$ I-CLINDE (26-28) confirmed literature data indicating that the TSPO is expressed during neuroinflammation (14) and indicated that ${ }^{123} \mathrm{I}$-CLINDE is a sensitive and specific tracer of astroglial activation.

The method of EAE induction that we used resulted in the development of 1 episode with full recovery, 1 relapse with full recovery, and 2 separate relapses. This heterogeneous pattern of clinical EAE gave us the opportunity to study and compare a variety of neurologic situations.

One of the most striking results, demonstrated by both in vivo biodistribution and PET imaging, was the strong TSPO expression observed in the CNS during the first disease episode, before the appearance of any clinical signs of EAE.

It is commonly thought that EAE in rodents progresses from caudal to rostral areas of the CNS. Although this may be the case for the spinal cord as a unit, it does not always hold true for the CNS because, as shown in mice with PLPinduced EAE, there is early (preclinical) blood-brain barrier breakdown, particularly in the cerebellum (29). Similar observations obtained with histochemical and MR imaging techniques have been reported $(3,30,31)$. The regional differences in the sensitivity of brain areas to upregulation of inflammatory molecules earlier or more rapidly (after encephalitogenic $\mathrm{T}$ cells producing a variety of proinflammatory molecules have passed through these areas) influence the susceptibility of different brain regions to neuroinflammation. Thus, the "easier access" for encephalitogenic lymphoblasts may explain the earlier glial activation and increased TSPO expression in the cerebellum.

With respect to the involvement of the olfactory bulbs early in the neuroinflammatory process, one should consider that the destiny of the mitotically active cells of the subventricular zone (SVZ) in adult rodents is to migrate to the olfactory bulbs, where they contribute to the replacement of granular and periglomerular neurons. In another model of RR EAE (induced with $\mathrm{MOG}_{35-55}$ in C57BL/6 mice) $(32,33)$, it was demonstrated that EAE enhanced the migration of SVZ-derived neural stem/progenitor cells (NSC) to the olfactory bulbs and that the adult mouse SVZ is a source of newly generated oligodendrocytes that may contribute, along with oligodendrocyte precursors, to the replacement of oligodendrocytes in inflammatory demyelinating diseases of the CNS. Because it is highly probable that the same would be valid for RR EAE in SJL/J mice, the activated NSC could then express the TSPO, which would reflect the increased uptake of ${ }^{18} \mathrm{~F}-\mathrm{PBR} 111$. Also, although morphologically mature mouse forebrain neurons are TSPO immunonegative, noninduced neural stem cells and neurons in the early stages of in vitro-induced differentiation can produce the TSPO (34).
FIGURE 5. PET study of displacement of ${ }^{18} \mathrm{~F}-\mathrm{PBR} 111$ uptake in brain at first EAE episode, with clinical score of 2, by PBR111. (A) Representative PET/CT images of ${ }^{18} \mathrm{~F}-\mathrm{PBR} 111$ uptake at 30-35 min after radiotracer injection. (B) Representative ${ }^{18} \mathrm{~F}$ PBR111 uptake in brain during displacement study. (C) Representative PET/CT images of ${ }^{18} \mathrm{~F}$-PBR111 uptake after injection of PBR111. \%ID = percentage injected dose.

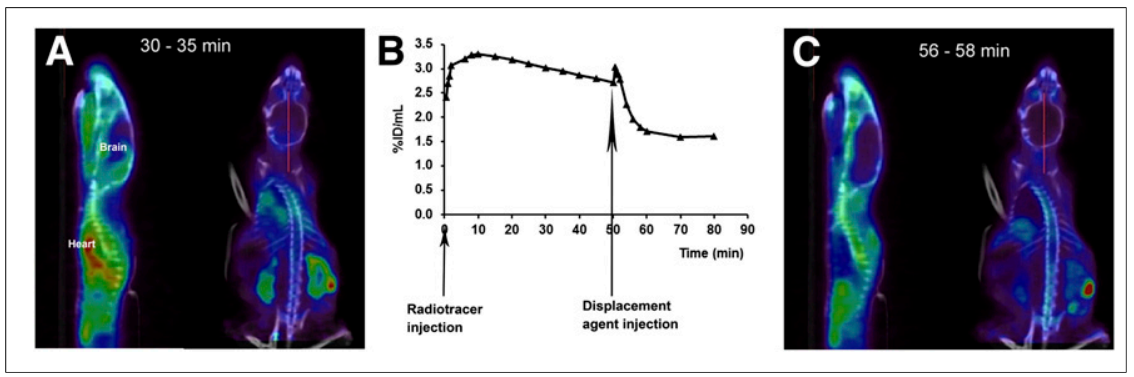




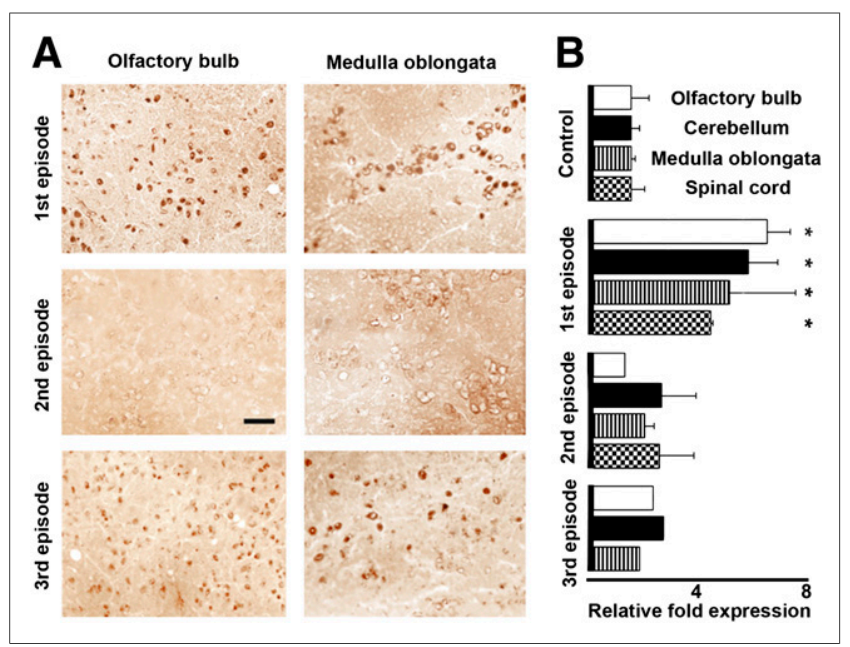

FIGURE 6. Dynamics of TSPO expression (as protein and messenger RNA) during 3 episodes of EAE induced by PLP-CFA. (A) Immunohistochemical labeling for TSPO. Bar, $25 \mu \mathrm{m}$. (B) RT PCR for TSPO in 4 brain areas. ${ }^{\star} \mathrm{P}<0.05$ for EAE vs. control.

With respect to PET imaging, our results for RR EAE parallel the PET imaging results for ethanol-induced injury in the rat striatum; that is, the extent of TSPO expression is dependent on the level of microglial activation (35).

The pattern of the relative TSPO values in different brain areas (reflecting the nonuniform distribution of microglia in these brain areas) was repeated during the 3 episodes. However, the strong increase in TSPO expression during the first EAE episode was followed by an absence of TSPO expression during the second episode and another elevation (as both messenger RNA and protein) during the third episode.

Recent reports of RR, primary-progressive, or secondary-progressive EAE models were compared with phases in the development of MS $(36,37)$. The involvement of

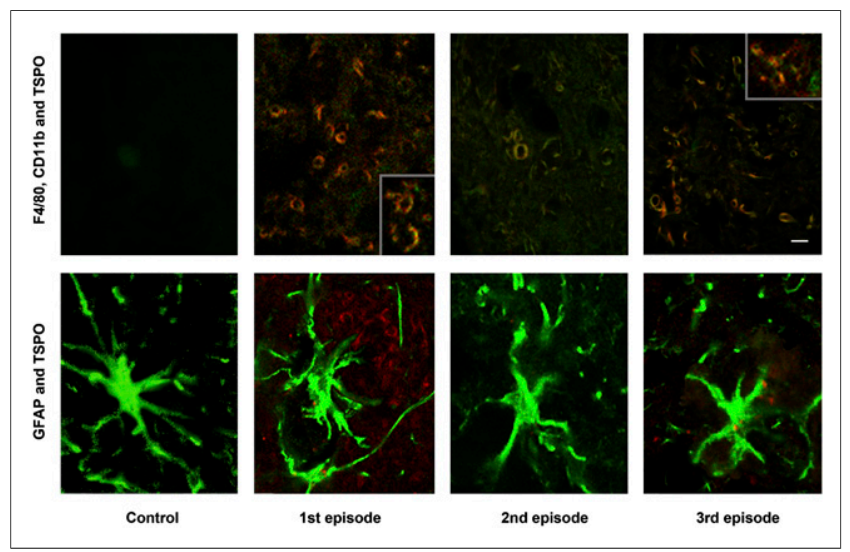

FIGURE 7. Double immunostaining for astrocytes and TSPO and for activated macrophages or microglial cells and TSPO during 3 episodes of PLP-induced EAE. (Top) Activated macrophages or microglial cells were labeled for F4/80 (green) or CD11b (green; inserts) and TSPO (red). Bar, $10 \mu \mathrm{m}$. (Bottom) Astrocytes were labeled for GFAP (green) and TSPO (red). multipotent NSC in CNS repair after injury was reviewed. NSC reside within specialized compartments or niches in the adult mammalian CNS, and the SVZ of the lateral ventricles in the brain is one such reservoir. During the acute phase of disease, NSC become activated and initiate regeneration but lose this ability during the chronic phases of disease, and a hypothesis was raised that chronic microglial activation contributes to the failure of NSC repair potential in the SVZ.

We also found fluctuations in the PET imaging results in the zone of the ventricles (Fig. 4), and the following values for the uptake of ${ }^{18} \mathrm{~F}-\mathrm{PBR} 111$ (compared with that in controls) were calculated: first episode, increased by $160 \%$; second episode, increased by $18 \%$; and third episode, increased by $100 \%$. One could speculate that, although there was a recovery from the neurologic symptoms after the first episode, the brain microglial cells had not fully "exited" the state of activation before the beginning of the second episode and chronic microglial activation contributed to the failure of NSC repair potential in the SVZ.

However, the situation was not repeated during the third episode. The aggravation of the clinical symptoms of EAE started about $1 \mathrm{wk}$ after the peak of the second episode. This finding could be attributed to the new notion that the activation of resident microglia contributes to both detrimental and protective aspects of the inflammatory process $(4,38)$. Thus, depending on the timing, brain microglia participates in inflammation (as in the first EAE episode), does not participate in inflammation (as in the second EAE episode), or participates again (as in the third EAE episode). The third episode started about $10 \mathrm{~d}$ after the neurologic score of the first episode returned to 0 . Ten days was also the time needed for TSPO expression to return to control values (Fig. 2B). Three markers of microglial activation (the TSPO, F4/80, and $\mathrm{CD} 11 \mathrm{~b}$ ) followed these dynamics, providing support for speculation that the brain microglia may also have a protective role during the second EAE episode.

In the spinal cord, TSPO expression was also statistically increased during the second episode, although to much lower levels than during the first episode. This discordance is now under investigation. To our knowledge, the results presented are the first to highlight the discrepancy between the clinical symptoms of EAE and TSPO expression in the brain, as measured by PET imaging at the peak of different EAE episodes. Some of these findings may be clarified in ongoing clinical trials involving ${ }^{18}$ F-PBR111 and microglial activation in patients with MS.

\section{CONCLUSION}

At the first episode of RR EAE, there was a large increase in TSPO expression in several brain areas and in the spinal cord. Importantly, during preclinical RR EAE, a significant increase in ${ }^{18} \mathrm{~F}-\mathrm{PBR} 111$ binding in all CNS areas (60\%-100\% above control values) suggests the potential use of TSPO screening for the early diagnosis of neuroinflammatory diseases with PET imaging. 


\section{DISCLOSURE}

The costs of publication of this article were defrayed in part by the payment of page charges. Therefore, and solely to indicate this fact, this article is hereby marked "advertisement" in accordance with 18 USC section 1734. This work was supported by NHMRC grant 418092. Maria Staykova is a Visiting Fellow at The John Curtin School of Medical Research, The Australian National University. No other potential conflict of interest relevant to this article was reported.

\section{ACKNOWLEDGMENTS}

The authors sincerely thank Dr. David Willenborg for his encouragement and advice. The authors thank Elaine Bean, Cathy Gillespie, Alexis Berard, Nicholas Howell, Emma Millard, and Kerynne Belbin for assistance with cryosections, the confocal microscope, imaging processing, and dissection.

\section{REFERENCES}

1. Imitola J, Chitnis T, Khoury SJ. Insights into the molecular pathogenesis of progression in multiple sclerosis: potential implications for future therapies. Arch Neurol. 2006;63:25-33.

2. Tsunoda I, Kuang LQ, Theil DJ, Fujinami RS. Antibody association with a novel model for primary progressive multiple sclerosis: induction of relapsing-remitting and progressive forms of EAE in H2s mouse strains. Brain Pathol. 2000;10:402418

3. Muller DM, Pender MP, Greer JM. Blood-brain barrier disruption and lesion localisation in experimental autoimmune encephalomyelitis with predominant cerebellar and brainstem involvement. J Neuroimmunol. 2005;160:162-169.

4. Gao Z, Tsirka SE. Animal models of MS reveal multiple roles of microglia in disease pathogenesis. Neurol Res Int. 2011;2011:383087.

5. Lublin FD, Reingold SC. Defining the clinical course of multiple sclerosis: results of an international survey-National Multiple Sclerosis Society (USA) Advisory Committee on Clinical Trials of New Agents in Multiple Sclerosis. Neurology. 1996;46:907-911.

6. Boretius S, Kasper L, Tammer R, Michaelis T, Frahm J. MRI of cellular layers in mouse brain in vivo. Neuroimage. 2009;47:1252-1260.

7. Chard D, Miller D. Is multiple sclerosis a generalized disease of the central nervous system? An MRI perspective. Curr Opin Neurol. 2009;22:214-218.

8. Barkhof F, Simon JH, Fazekas F, et al. MRI monitoring of immunomodulation in relapse-onset multiple sclerosis trials. Nat Rev Neurol.. 2012;8:13-21.

9. Papadopoulos V, Baraldi M, Guilarte TR, et al. Translocator protein (18 KDa): new nomenclature for the peripheral-type benzodiazepine receptor based on its structure and molecular function. Trends Pharmacol Sci. 2006;27:402-409.

10. Venneti S, Wang GJ, Nguyen J, Wiley CA. The positron emission tomography ligand DAA1106 binds with high affinity to activated microglia in human neurological disorders. J Neuropathol Exp Neurol. 2008;67:1001-1010.

11. Papadopoulos V, Lecanu L, Brown RC, Han Z, Yao ZX. Peripheral-type benzodiazepine receptor in neurosteroid biosynthesis, neuropathology and neurological disorders. Neuroscience. 2006;138:749-756.

12. Casellas P, Galiegue S, Basile AS. Peripheral benzodiazepine receptors and mitochondrial function. Neurochem Int. 2002;40:475-486.

13. Chen MK, Guilarte TR. Translocator protein $18 \mathrm{kDa}$ (TSPO): molecular sensor of brain injury and repair. Pharmacol Ther. 2008;118:1-17.

14. Ji B, Maeda J, Sawada M, et al. Imaging of peripheral benzodiazepine receptor expression as biomarkers of detrimental versus beneficial glial responses in mouse models of Alzheimer's and other CNS pathologies. J Neurosci. 2008;28:1225512267.

15. Oh U, Fujita M, Ikonomidou VN, et al. Translocator protein PET imaging for glial activation in multiple sclerosis. J Neuroimmune Pharmacol. 2011;6:354-361.

16. Venneti S, Lopresti BJ, Wiley CA. The peripheral benzodiazepine receptor (translocator protein $18 \mathrm{KDa}$ ) in microglia: from pathology to imaging. Prog Neurobiol. 2006;80:308-322.
17. Versijpt JJ, Dumont F, Vanlaere KJ, et al. Assessment of neuroinflammation and microglial activation in Alzheimer's disease with radiolabelled PK11195 and single photon emission computed tomography: a pilot study. Eur Neurol. 2003;50:39-47.

18. Gerhard A, Banati RB, Goerres GB, et al. [C-11](R)-PK11195 PET imaging of microglial activation in multiple system atrophy. Neurology. 2003;61:686-689.

19. Chauveau F, Boutin H, Van Camp N, Dolle F, Tavitian B. Nuclear imaging of neuroinflammation: a comprehensive review of [C-11]PK11195 challengers. Eur J Nucl Med Mol Imaging. 2008;35:2304-2319.

20. Fookes CJ, Pham TQ, Mattner F, et al. Synthesis and biological evaluation of substituted $\left[{ }^{18} \mathrm{~F}\right]$ imidazo $[1,2-\mathrm{a}]$ pyridines and $\left[{ }^{18} \mathrm{~F}\right]$ pyrazolo[1,5-a]pyrimidines for the study of the peripheral benzodiazepine receptor using positron emission tomography. J Med Chem. 2008;51:3700-3712.

21. Van Camp N, Boisgard R, Kuhnast B, et al. In vivo imaging of neuroinflammation: a comparative study between [F-18]PBR111, [C-11]CLINME and [C-11] PK11195 in an acute rodent model. Eur J Nucl Med Mol Imaging. 2010;37: 962-972.

22. Bourdier T, Pham TQ, Henderson D, et al. Automated radiosynthesis of $\left.{ }^{18} \mathrm{~F}\right]$ PBR111 and $\left[{ }^{18} \mathrm{~F}\right]$ PBR102 using the Tracerlab FXFN and Tracerlab MXFDG module for imaging the peripheral benzodiazepine receptor with PET. Appl Radiat Isot. 2012;70:176-183.

23. Disselhorst JA, Brom M, Laverman P, et al. Image-quality assessment for several positron emitters using the NEMA NU 4-2008 standards in the Siemens Inveon small-animal PET scanner. J Nucl Med. 2010;51:610-617.

24. BrainVISA/Anatomist Web page. Available at: http://brainvisa.info. Accessed December 28, 2012.

25. Ne MRMAT (Neurological Atlas) Mouse Brain Database Web page. Available at: http://www.bnl.gov/medical/RCIBI/mouse/. Accessed December 28, 2012.

26. Mattner F, Katsifis A, Staykova M, Ballantyne P, Willenborg DO. Evaluation of a radiolabelled peripheral benzodiazepine receptor ligand in the central nervous system inflammation of experimental autoimmune encephalomyelitis: a possible probe for imaging multiple sclerosis. Eur J Nucl Med Mol Imaging. 2005;32: 557-563.

27. Mattner F, Staykova M, Callaghan P, et al. Assessment of neuroinflammation in transferred EAE via a translocator protein ligand. In: Weissert R, ed. Experimental Autoimmune Encephalomyelitis: Models of Disease Biology and Experimental Therapy. Rijeka, Croatia: InTech; 2012:47-64.

28. Mattner F, Bandin DL, Staykova M, et al. Evaluation of $\left[{ }^{123} \mathrm{I}\right]-\mathrm{CLINDE}$ as a potent SPECT radiotracer to assess the degree of astroglia activation in cuprizone-induced neuroinflammation. Eur J Nucl Med Mol Imaging. 2011;38: 1516-1528.

29. Tonra JR, Reiseter BS, Kolbeck R, et al. Comparison of the timing of acute blood-brain barrier breakdown to rabbit immunoglobulin $\mathrm{G}$ in the cerebellum and spinal cord of mice with experimental autoimmune encephalomyelitis. J Comp Neurol. 2001;430:131-144.

30. Wuerfel J, Tysiak E, Prozorovski T, et al. Mouse model mimics multiple sclerosis in the clinico-radiological paradox. Eur J Neurosci. 2007;26:190-198.

31. Lees JR, Golumbek PT, Sim J, Dorsey D, Russell JH. Regional CNS responses to IFN-gamma determine lesion localization patterns during EAE pathogenesis. J Exp Med. 2008;205:2633-2642.

32. Sajad M, Zargan J, Chawla R, Umar S, Khan HA. Upregulation of CSPG3 accompanies neuronal progenitor proliferation and migration in EAE. $J \mathrm{Mol}$ Neurosci. 2011;43:531-540.

33. Picard-Riera N, Decker L, Delarasse C, et al. Experimental autoimmune encephalomyelitis mobilizes neural progenitors from the subventricular zone to undergo oligodendrogenesis in adult mice. Proc Natl Acad Sci USA. 2002;99:1321113216.

34. Varga B, Marko K, Hadinger N, et al. Translocator protein (TSPO 18KDa) is expressed by neural stem and neuronal precursor cells. Neurosci Lett. 2009;462: 257-262.

35. Ito F, Toyama H, Kudo G, et al. Two activated stages of microglia and PET imaging of peripheral benzodiazepine receptors with [C-11]PK11195 in rats. Ann Nucl Med. 2010;24:163-169.

36. Rasmussen S, Imitola J, Ayuso-Sacido A, et al. Reversible neural stem cell niche dysfunction in a model of multiple sclerosis. Ann Neurol. 2011;69:878-891.

37. Pluchino S, Muzio L, Imitola J, et al. Persistent inflammation alters the function of the endogenous brain stem cell compartment. Brain. 2008;131: 2564-2578.

38. Hanisch UK, Kettenmann H. Microglia: active sensor and versatile effector cells in the normal and pathologic brain. Nat Neurosci. 2007;10:1387-1394. 